\title{
Note about null dimensional reduction of M5-brane
}

\author{
Josef Klusoň* \\ Department of Theoretical Physics and Astrophysics, Faculty of Science, Masaryk University, \\ Kotlářská 2, 61137 Brno, Czech Republic
}

(Received 2 July 2021; accepted 11 January 2022; published 26 January 2022)

\begin{abstract}
In this short note we study null dimensional reduction of M5-brane covariant action. We analyze longitudinal dimensional reduction that leads to nonrelativistic D4-brane and transverse reduction that leads to NS5-brane in nonrelativistic string theory.
\end{abstract}

DOI: 10.1103/PhysRevD.105.026027

\section{INTRODUCTION AND SUMMARY}

It has been known from the late 1990s that string theories have origin in eleven dimensional theory: M-theory [1]. Since all string theories can be considered as limits of M-theory it is clear that M-theory has to contain extended objects that are parent objects of fundamental strings, D-branes and NS5-branes [2], for review see for example [3]. The fundamental object of M-theory is M2-brane as string is fundamental for string theory since M2-brane electrically couples to three form $C^{(3)}$ in the same way as string couples to two form $B^{(2)}$. On the other hand, a more interesting object in M-theory is M5-brane [4-10]. This is a six dimensional object with an exceptional property that on its world-volume self-dual three form field propagates that cannot be described in Lagrangian formalism. Despite this fact, covariant action for M5-brane was proposed and extensively studied in [4-6]. In particular, it was shown in [4] that this M5-brane dimensionally reduces into a D4-brane under double dimensional reduction and it was also shown in [11] that D3-brane action arises through double dimensional reduction of M5-brane action on torus [11].

In order to get more information about M5-brane covariant action it would be certainly interesting to study its properties in a different background. Such an example could be analysis of M5-brane action under null dimensional reduction of M-theory. It is well known that null dimensional reduction is rather subtle in the case of the gravity action $[12,13]$ however it can be performed on the level of equations of motion. Further, it is also well known that null dimensional reduction of gravity leads to torsional

\footnotetext{
*klu@physics.muni.cz
}

Published by the American Physical Society under the terms of the Creative Commons Attribution 4.0 International license. Further distribution of this work must maintain attribution to the author(s) and the published article's title, journal citation, and DOI. Funded by SCOAP ${ }^{3}$.
Newton-Cartan gravity $[13,14]$ in lower dimensional spacetime. Clearly we can deduce that null dimensional reduction of M-theory would lead to torsional Newton-Cartan string theory even if the precise analysis of this problem has not been performed yet. ${ }^{1}$ On the other hand, it is possible to ask the question how extended objects in M-theory map under null dimensional reductions. In our previous paper [18] we studied null dimensional reduction of the M2-brane. We showed that when we have M2-brane wrapped null direction we get fundamental string action in torsional Newton-Cartan string theory. Further, when we considered M2-brane transverse to this null direction we got D2-brane action in the same background. Then we can ask the question how this procedure can be applied in the case of the M5-brane. Thee goal of this paper is to address this problem. We first review dimensional reduction along a spacelike circle that should lead to D4-brane in type IIA theory. This procedure was first discussed in [4] but we analyze this problem in more detail and in a slightly different way. First of all we perform spatial dimensional reduction of the M5-brane that leads to five dimensional action with a propagating two form on its world volume. Since two form in five dimensions is dual to one form it is natural to perform duality transformations following $[8,19]$. We show that dual action for dimensional reduced M5-brane action is D4-brane action in type IIA theory. We would like to stress that this analysis could be considered as dual to the analysis performed in [8] where the starting point was D4-brane and then it was shown that dual action corresponds to M5-brane action dimensionally reduced along spatial dimension.

As the next step we extend this idea to the case of null dimensional reduction of the M5-brane. We start with the discussion of the background with null isometry following $[12,13]$. Since it is not completely clear how to perform null dimensional reduction in the case of three form $C^{(3)}$ that

\footnotetext{
${ }^{1}$ See also [15]. For another study of nonrelativistic limits of M-theory, see [16,17].
} 
appears as additional background field in M-theory we restrict ourselves to the case when this form vanishes. Then we insert this background metric into covariant M5-brane action and we obtain its dimensionally reduced form. Again since this action still depends on a two form field it is natural to perform its duality transformation. Now we should be more careful than in the case of the relativistic M5-brane since the induced metric corresponds to a Newton-Cartan metric where there is no Lorentz invariance in the target space-time. For that reason we consider a more general situation with diagonal components of spatial metric $h^{\alpha \beta}$, nonzero component of time one form $\tau_{\alpha}$ together with nonzero components of $m_{\alpha}$ where the meaning of these symbols will be given below. Then we will be able to perform duality transformation as in the case of the spatial dimensional reduction and we argue that the resulting action corresponds to a D4-brane in the torsional Newton-Cartan background. We also perform transverse null isometry reduction of the M5-brane and we argue that resulting action corresponds to the NS5-brane in the nonrelativistic type IIA theory even if the resulting action is very complicated.

Let us outline our results and suggest possible extension of this work. We find an action for the D4-brane in torsional Newton-Cartan type IIA theory. This is the main result of this paper that together with the paper [18] proves that extended objects in M-theory map under null dimensional reduction to fundamental strings an D-branes in torsional Newton-Cartan string theory. It is remarkable that these actions have functionally the same form as their relativistic counterparts since they depend on the pullback of the Newton-Cartan volume form [14]. On the other hand, this fact raises a very important question which is a relation between D4-brane action derived in this paper and the form of nonrelativistic Dp-brane in torsional Newton-Cartan (NC) background as was found in [20,21]. In these papers the nonrelativistic Dp-brane in torsional NC background was found by T-duality from relativistic $\mathrm{D}(\mathrm{p}+1)$-brane in the background with lightlike isometry when this $\mathrm{D}(\mathrm{p}+1)$-brane is extended along the lightlike dimension and we perform T-duality along it. ${ }^{2}$ This procedure is an extension of the definition of torsional NC string [20,29-33] to the case of higher dimensional objects. It would be certainly very interesting to understand how these apparently different actions for nonrelativistic Dp-branes in torsional nonrelativistic string theories are related.

This paper is organized as follows. In Sec. II we review the main properties of M5-brane action and perform its dimensional reduction along the spatial dimension. In Sec. III we perform dimensional reduction when the M5-brane wraps null direction. Finally in Sec. IV we briefly mention dimensional reduction of the M5-brane

\footnotetext{
${ }^{2}$ Another form of nonrelativistic D4-brane actions was derived by a specific limiting procedure [22-25]; see also [26-28].
}

when it is transverse to the null direction. The resulting action should correspond to a NS5-brane in torsional Newton-Cartan string theory as transverse dimensional reduction of the M5-brane along a spatial circle corresponds to a NS5-brane in type IIA theory.

\section{COVARIANT M5-BRANE ACTION AND SPATIAL DIMENSIONAL REDUCTION}

In this section we introduce basic facts about covariant M5-brane action and study its spatial dimensional reduction. The covariant form of M5-brane action was introduced in [4-7]; see also [8,9]. Explicitly, we consider M5-brane action in the form ${ }^{3}$

$$
\begin{aligned}
S= & -T_{M 5} \int d^{6} \xi\left[\sqrt{-\operatorname{det}\left(\hat{g}_{m n}+i \frac{\hat{H}_{m n}^{*}}{\sqrt{\widehat{\partial a \partial} a}}\right)}\right. \\
& \left.-\frac{\sqrt{-\hat{g}}}{4 \widehat{\partial a \partial a}} \hat{H}^{* m n} H_{m n r} \partial^{r} a\right],
\end{aligned}
$$

where

$$
\widehat{\partial a \partial a}=\partial_{m} a \hat{g}^{m n} \partial_{n} a
$$

and where

$$
m, \quad n=0,1, \ldots, 5
$$

are vector indices of $d=6$ world-volume coordinates $\xi^{m}$,

$$
M, \quad N=0,1, \ldots, 10
$$

are vector indices of $D=11$ target space-time coordinates $X^{M}$

$$
\hat{g}_{m n}=\partial_{m} X^{M} \hat{g}_{M N} \partial_{n} X^{N}
$$

is the world-volume metric that is induced by embedding the five-brane into $D=11$ background with metric $\hat{g}_{M N}$. Further, we have

$$
\hat{H}_{m n}^{*}=\frac{1}{6 \sqrt{-\hat{g}}} \hat{g}_{m r} \hat{g}_{n s} \epsilon^{r s t u v w} H_{t u v} \partial_{w} a,
$$

where $H_{m n l}$ is the field strength for the world-volume antisymmetric tensor $B_{m n}$ :

$$
H_{m n l}=\partial_{l} B_{m n}+\partial_{m} B_{n l}+\partial_{n} B_{l m} .
$$

Finally $a$ is the world-volume scalar field that ensures the covariance of the model.

\footnotetext{
${ }^{3}$ We work with the metric of the signature $(-,+, \cdots,+)$.
} 
The M5-brane action is invariant under the following gauge symmetries [4]. The first one is usual gauge symmetry,

$$
\delta a=0, \quad \delta B_{m n}=\partial_{m} \phi_{n}-\partial_{n} \phi_{m} .
$$

There is another nontrivial gauge symmetry in the form [4-6]

$$
\delta B_{m n}=\phi_{m} \partial_{n} a-\partial_{m} a \phi_{n}, \quad \delta a=0 .
$$

Finally the action is invariant under transformation [4-6]

$$
\delta a=\phi, \quad \delta B_{m n}=\frac{\delta a}{\sqrt{\widehat{\partial a \partial a}}}\left[\hat{H}^{*}{ }_{m n}-H_{m n p} \hat{g}^{p s} \frac{\partial_{s} a}{\sqrt{\widehat{\partial a \partial a}}}\right],
$$

where

$$
\begin{aligned}
\hat{\mathcal{H}}_{m n}^{*} & =-\frac{2}{\sqrt{-\hat{g}}} \frac{\delta \mathcal{L}_{\mathrm{DBI}}}{\delta \hat{H}^{* m n}}, \\
\mathcal{L}_{\mathrm{DBI}} & =\sqrt{-\operatorname{det}\left(\hat{g}_{m n}+i \frac{\hat{H}_{m n}^{*}}{\sqrt{\widehat{\partial a \partial a}}}\right)} .
\end{aligned}
$$

The symmetries (9) and (10) are fundamental properties of M5-brane action (1). They ensure that $B_{m n}$ equations of motion reduce to self-duality conditions, see [4-6] for more details. As was shown in [4] the equation of motion for $a(\xi)$ is a consequence of the equations of motion for $B_{m n}$ and hence it is not a new field equation. As a result $a$ can be directly eliminated by fixing the gauge transformations (9). Following [4] we fix $a$ by imposing condition

$$
\partial_{m} a=\delta_{m}^{5} .
$$

Then using (9) we get

$$
\delta B_{5 m}=\frac{1}{2} \phi_{m}
$$

and hence we can fix components of $B_{5 m}$ to be equal to zero:

$$
B_{5 m}=0 .
$$

We impose this condition in case of spatial and null dimensional reduction of the M5-brane.

Before we proceed to the case of null dimensional reduction we review spatial dimensional reduction of M5-brane. To do this we consider the eleven dimensional line element in the form

$$
d s^{2}=e^{-\frac{2}{3} \phi} g_{\mu \nu} d x^{\mu} d x^{\nu}+e^{\frac{4}{3} \phi}\left(d y-C_{\mu} d x^{\mu}\right)^{2},
$$

where $\mu, \nu=0,1, \ldots, 9$. We impose spatial dimensional reduction when we presume that $y$ coincides with $\xi^{5}$ and when all world-volume fields do not depend on $\xi^{5}$ except $a$ that obeys the condition (12). Let us denote the remaining world-volume coordinates as $\xi^{\alpha}, \alpha, \beta, \gamma, \ldots=0,1, \ldots, 4$. Then using (14) and also the fact that all world-volume fields do not depend on $\xi^{5}$ we find that there are nonzero components of $H_{\alpha \beta \gamma}$ only.

Then with the help of (15) we find that components of the induced metric are equal to

$\hat{g}_{\alpha \beta}=\partial_{\alpha} x^{\mu} \hat{g}_{\mu \nu} \partial_{\beta} x^{\nu}=e^{-\frac{2}{3} \phi} g_{\alpha \beta}+e^{\frac{4}{3} \phi} C_{\alpha} C_{\beta}$,

$\hat{g}_{\alpha y}=-e^{\frac{4}{3} \phi} C_{\alpha}, \quad \hat{g}_{y y}=e^{\frac{4}{3} \phi}$

with corresponding components of the inverse metric $\hat{g}^{m n}$ :

$\hat{g}^{\alpha \beta}=e^{\frac{2}{3} \phi} g^{\alpha \beta}, \quad \hat{g}^{y \alpha}=e^{\frac{2}{3} \phi} C_{\gamma} g^{\gamma \beta}, \quad \hat{g}^{y y}=e^{-\frac{4}{3} \phi}+e^{\frac{2}{3} \phi} C_{\alpha} g^{\alpha \beta} C_{\beta}$,

where $g_{\alpha \beta}$ is the induced five dimensional matrix and hence $g^{\alpha \beta}$ is its inverse metric that obeys the equation

$$
g_{\alpha \beta} g^{\beta \gamma}=\delta_{\alpha}^{\gamma}
$$

Further, using (16) we obtain

$$
\operatorname{det} \hat{g}_{m n}=e^{-2 \phi} \operatorname{det} g_{\alpha \beta} .
$$

Let us now turn our attention to $\hat{H}_{m n}^{*}$ that using (12) has the form

$$
\hat{H}_{m n}^{*}=\frac{1}{6 \sqrt{-\hat{g}}} \hat{g}_{m r} \hat{g}_{n s} \epsilon^{r s t u v 5} H_{t u v} .
$$

Now since $\epsilon^{m n r s t u}$ is a totally antisymmetric symbol we find that all nonzero components have the form $\epsilon^{\alpha \beta \gamma \delta \omega 5} \equiv \epsilon^{\alpha \beta \gamma \delta \omega}$ so that

$$
\hat{H}_{m n}^{*}=\frac{e^{-2 \phi}}{6 \sqrt{-g}} \hat{g}_{m \alpha} \hat{g}_{n \beta} \epsilon^{\alpha \beta \gamma \delta \omega} H_{\gamma \delta \omega} .
$$

To proceed further we use the fact that we can write

$$
\begin{aligned}
& \sqrt{-\operatorname{det}\left(\hat{g}_{m n}+i \frac{\hat{H}_{m n}^{*}}{\sqrt{\widehat{\partial a \partial a}}}\right)} \\
& =\sqrt{-\operatorname{det} \hat{g}_{m k}} \sqrt{-\operatorname{det}\left(\hat{g}^{k l}+i \frac{\hat{g}^{k r} \hat{H}_{r s}^{*} \hat{g}^{s l}}{\sqrt{\widehat{\partial a \partial a}}}\right)} \sqrt{-\operatorname{det} \hat{g}_{l n}},
\end{aligned}
$$

where 


$$
\hat{g}^{k l}+i \frac{\hat{g}^{k r} \hat{H}_{r s}^{*} \hat{g}^{s l}}{\sqrt{\widehat{\partial a \partial a}}}=\hat{g}^{k l}+i \frac{1}{6 \sqrt{-\hat{g}} \sqrt{\widehat{\partial a \partial a}}} \epsilon^{k l t u v w} H_{t u v} \partial_{w} a .
$$

Now in the gauge $\partial_{m} a=\delta_{m}^{5}$ we obtain

$$
\begin{aligned}
& \operatorname{det}\left(\hat{g}^{k l}+i \frac{1}{6 \sqrt{-\hat{g}} \sqrt{\widehat{\partial a \partial a}}} e^{k l t u v w} H_{\text {tuv }} \partial_{w} a\right) \\
& =\left|\begin{array}{cc}
e^{\frac{2}{3} \phi} g^{\alpha \beta}+i \frac{1}{6 \sqrt{-\hat{g}} \sqrt{\widehat{\partial a \partial a}}} e^{\alpha \beta \gamma \delta \omega} H_{\gamma \delta \omega} & g^{\alpha \gamma} C_{\gamma} e^{\frac{2}{3} \phi} \\
C_{\gamma} g^{\gamma \beta} e^{\frac{2}{3} \phi} & e^{-\frac{4}{3} \phi}+e^{\frac{2}{3} \phi} C_{\alpha} g^{\alpha \beta} C_{\beta}
\end{array}\right| \\
& =\operatorname{det}\left(e^{\frac{2}{3} \phi} g^{\alpha \beta}-\frac{e^{\frac{4}{3} \phi} g^{\alpha \gamma} C_{\gamma} C_{\delta} g^{\delta \beta}}{e^{-\frac{4}{3} \phi}+e^{\frac{2}{3} \phi} C_{\alpha} g^{\alpha \beta} C_{\beta}}+i \frac{1}{6 e^{-\frac{5}{3} \phi} \sqrt{-\operatorname{det} g} \sqrt{1+e^{2 \phi} C_{\alpha} g^{\alpha \beta} C_{\beta}}} \epsilon^{\alpha \beta \gamma \delta \omega} H_{\gamma \delta \omega}\right) \\
& \\
& \times\left(e^{-\frac{4}{3} \phi}+e^{\frac{2}{3} \phi} C_{\alpha} g^{\alpha \beta} C_{\beta}\right) .
\end{aligned}
$$

Let us now analyze the second term in the action (1) that using (12) takes the form

$$
\begin{aligned}
-\frac{\sqrt{-\hat{g}}}{4 \partial \widehat{a \partial a}} \hat{H}^{* m n} H_{m n r} \partial^{r} a & =-\frac{\sqrt{-\hat{g}}}{4 \hat{g}^{y y}} \hat{H}^{* \alpha \beta} H_{\alpha \beta \gamma} \hat{g}^{\gamma 5} \\
& =-\frac{e^{\frac{2}{3} \phi}}{24\left(e^{-\frac{4}{3} \phi}+e^{\frac{2}{3} \phi} C_{\alpha} C^{\alpha}\right)} \epsilon^{\alpha \beta \gamma \delta \omega} H_{\gamma \delta \omega} H_{\alpha \beta \rho} g^{\rho \sigma} C_{\sigma} .
\end{aligned}
$$

Collecting these terms together we finally obtain the dimensionally reduced form of the action:

$$
\begin{aligned}
S= & -T_{D 4} \int d^{5} \xi e^{-\phi} \sqrt{-\operatorname{det}\left(g_{\alpha \beta}-\frac{C_{\alpha} C_{\beta}}{1+e^{2 \phi} C_{\gamma} g^{\gamma \delta} C_{\delta}}+i \frac{e^{\phi} g_{\alpha \alpha^{\prime}} g_{\beta \beta^{\prime}}}{6 \sqrt{-\operatorname{det} g} \sqrt{1+e^{2 \phi} C_{\gamma} g^{\gamma \delta} C_{\delta}}} \epsilon^{\alpha^{\prime} \beta^{\prime} \gamma \delta \omega} H_{\gamma \delta \omega}\right)} \\
& \times \sqrt{1+e^{2 \phi} C_{\gamma} g^{\gamma \delta} C_{\delta}} \\
& -\frac{e^{2 \phi}}{24\left(1+e^{2 \phi} C_{\gamma} g^{\gamma \delta} C_{\delta}\right)} \epsilon^{\alpha \beta \gamma \delta \omega} H_{\gamma \delta \omega} H_{\alpha \beta \rho} g^{\rho \sigma} C_{\sigma} \\
& +\frac{1}{6} \int d^{5} \xi V^{\alpha \beta \gamma}\left(H_{\alpha \beta \gamma}-\left(\partial_{\alpha} B_{\beta \gamma}+\partial_{\beta} B_{\gamma \alpha}+\partial_{\gamma} B_{\alpha \beta}\right)\right),
\end{aligned}
$$

where we identified $T_{D 4} \equiv T_{M 5} \int d y$ since we anticipate that the action above corresponds to D4-brane action in type IIA theory. However this correspondence is not quite correct since the action (26) still depends on the field strength of two form field $B_{\alpha \beta}$ while Dirac-Born-Infeld (DBI) action is defined using gauge field which is one form. In order to find such an action we should perform duality transformation following $[8,19]$. The first step is to extend the action as in (26) that allows us to treat $B_{\beta \gamma}$ and $H_{\alpha \beta \gamma}$ as independent variables. Since now $B_{\alpha \beta}$ is independent it can be integrated out which implies

$$
\partial_{\alpha} V^{\alpha \beta \gamma}=0
$$

This equation has a solution in five dimensions in the form

$$
V^{\alpha \beta \gamma}=\epsilon^{\alpha \beta \gamma \delta \omega} \partial_{\delta} A_{\omega}
$$

In order to find dual action we should integrate out three form $H_{\alpha \beta \gamma}$. To do this we will perform an inverse procedure to the analysis presented in $[8,19]$. Explicitly, we start with the action (26) and find its dual formulation. Following $[8,19]$ we use five dimensional Lorentz invariance and switch to the local inertial frame where $g_{\alpha \beta}=\eta_{\alpha \beta}$ and where we also presume that $C_{\alpha}$ has nonzero component $C_{0}$ only. We further presume that there are nonzero components $H_{340}$ and $H_{120}$ so that the matrix 


$$
\mathbf{M}_{\alpha \beta} \equiv g_{\alpha \beta}-\frac{e^{2 \phi} C_{\alpha} C_{\beta}}{1+e^{2 \phi} C_{\gamma} C^{\gamma}}+i \frac{e^{\phi} g_{\alpha \alpha^{\prime}} g_{\beta \beta^{\prime}}}{6 \sqrt{-\operatorname{det} g} \sqrt{1+e^{2 \phi} C_{\gamma} C^{\gamma}}} \epsilon^{\alpha^{\prime} \beta^{\prime} \gamma \delta \omega} H_{\gamma \delta \omega}
$$

has the form

$$
\mathbf{M}_{\alpha \beta}=\left(\begin{array}{ccccc}
-1-\frac{e^{2 \phi} C_{0}^{2}}{1-e^{2 \phi} C_{0}^{2}} & 0 & 0 & 0 & 0 \\
0 & 1 & i \frac{e^{\phi}}{\sqrt{1-e^{2 \phi} C_{0}^{2}}} H_{340} & 0 & 0 \\
0 & -i \frac{e^{\phi}}{\sqrt{1-e^{2 \phi} C_{0}^{2}}} H_{340} & 1 & 0 & 0 \\
0 & 0 & 0 & 1 & i \frac{e^{\phi}}{\sqrt{1-e^{2 \phi} C_{0}^{2}}} H_{120} \\
0 & 0 & 0 & -i \frac{e^{\phi}}{\sqrt{1-e^{2 \phi} C_{0}^{2}}} H_{120} & 1
\end{array}\right)
$$

so that $\operatorname{det} \mathbf{M}_{\alpha \beta}$ is equal to

$$
\operatorname{det} \mathbf{M}_{\alpha \beta}=-\left(1+\frac{e^{2 \phi} C_{0}^{2}}{1-e^{2 \phi} C_{0}^{2}}\right)\left(1-\frac{e^{2 \phi}}{1-e^{2 \phi} C_{0}^{2}} H_{2}^{2}\right)\left(1-\frac{e^{2 \phi}}{1-e^{2 \phi} C_{0}^{2}} H_{1}^{2}\right),
$$

where $H_{340}=H_{2}, H_{120}=H_{1}$. We also introduce notation $V^{120}=V_{1}, V^{340}=V_{2}$. Then the action has the form

$$
\begin{aligned}
S= & -T_{D 4} \int d^{5} \xi e^{-\phi} \sqrt{\left(1-\frac{e^{2 \phi}}{1-e^{2 \phi} C_{0}^{2}} H_{2}^{2}\right)\left(1-\frac{e^{2 \phi}}{1-e^{2 \phi} C_{0}^{2}} H_{1}^{2}\right)} \\
& +T_{D 4} \int d^{5} \xi\left(\frac{e^{2 \phi} C_{0}}{1-e^{2 \phi} C_{0}^{2}} H_{1} H_{2}+V_{1} H_{1}+V_{2} H_{2}\right) .
\end{aligned}
$$

Let us solve equations of motion for $H_{1}$ and $H_{2}$ that follow from (32)

$$
\begin{aligned}
& \Delta e^{-\phi} \sqrt{\frac{1-\Delta H_{2}^{2}}{1-\Delta H_{1}^{2}}} H_{1}+\Delta C_{0} H_{2}+V_{1}=0, \\
& \Delta e^{-\phi} \sqrt{\frac{1-\Delta H_{1}^{2}}{1-\Delta H_{2}^{2}}} H_{2}+\Delta C_{0} H_{1}+V_{2}=0,
\end{aligned}
$$

where

$$
\Delta=\frac{e^{2 \phi}}{1-e^{2 \phi} C_{0}^{2}}
$$

The Eqs. (33) have solutions

$$
H_{1}=V_{2} C_{0} \mp e^{-\phi} \sqrt{\frac{1+V_{2}^{2}}{1+V_{1}^{2}}} V_{1}, \quad H_{2}=V_{1} C_{0} \mp e^{-\phi} \sqrt{\frac{1+V_{1}^{2}}{1+V_{2}^{2}}} V_{2} .
$$

Finally inserting (35) into (32) we obtain

$$
S=-T_{D 4} \int d^{5} \xi\left[e^{-\phi} \sqrt{\left(1+V_{1}^{2}\right)\left(1+V_{2}^{2}\right)}-C_{0} V_{1} V_{2}\right],
$$

where we used the fact that multiplication of two equations given in (33) leads to the result 


$$
\begin{aligned}
- & e^{-\phi} \sqrt{\left(1-\Delta H_{1}^{2}\right)\left(1-\Delta H_{2}^{2}\right)} \\
& =\frac{1}{\Delta H_{1}}\left(\Delta C_{0} H_{2}+V_{1}\right)\left(1-\Delta H_{1}^{2}\right)
\end{aligned}
$$

and we have chosen the-sign in (35) to have an action with correct sign. Then we finally introduce $F_{\alpha \beta}=\partial_{\alpha} A_{\beta}-\partial_{\beta} A_{\alpha}$ and restore world-volume covariance so that the action (36) has the final form

$$
\begin{aligned}
S= & -T_{D 4} \int d^{5} \xi\left[e^{-\phi} \sqrt{-\operatorname{det}\left(g_{\alpha \beta}+F_{\alpha \beta}\right)}\right. \\
& \left.-\frac{1}{8} \epsilon^{\alpha \beta \gamma \delta \omega} C_{\alpha} F_{\beta \gamma} F_{\delta \omega}\right] .
\end{aligned}
$$

This is action for the D4-brane in the background with metric $g_{\mu \nu}$ and Ramond-Ramond form $C_{\mu}$. Observe that we proceeded in the inverse direction to the duality transformation studied in [8] where the starting point was the D4-brane in the background with nontrivial metric and Ramond-Ramond one form. Finally note also that the analysis performed in this section could be easily generalized to the case of nontrivial background three form $C^{(3)}$, following $[4,8]$. However we are not going to discuss this generalization in this section since we will also consider zero three form when we perform null dimensional reduction of the M5-brane.

\section{NULL DIMENSIONAL REDUCTION OF M5-BRANE}

We begin this section with the review of basic facts about null dimensional reduction of gravity.

Let us consider gravity background with null isometry and perform its dimensional reduction, following $[12,13]$. We presume that we have vierbein $\hat{E}_{M}{ }^{A}$ and inverse $\hat{E}^{M}{ }_{A}$ where $M=0,1, \ldots, 10$ and tangent space indices $A, B=$ $0,1, \ldots, 10$ that obey

$$
\hat{E}_{M}{ }^{A} \hat{E}^{M}{ }_{B}=\delta_{B}^{A}, \quad \hat{E}_{M}{ }^{A} \hat{E}^{N}{ }_{A}=\delta_{M}^{N} .
$$

If we try to dimensionally reduce along null direction we assume an existence of null Killing vector $\xi=\xi^{M} \partial_{M}$ that obeys

$$
\mathcal{L}_{\xi} \hat{g}_{M N}=0, \quad \xi^{M} \hat{g}_{M N} \xi^{N}=0 .
$$

Without loss of generality we choose adapted coordinates $x^{M}=\left(x^{\mu}, y\right)$ where $\mu=0,1, \ldots, 9$ and where $x^{10}=y$ so that $\xi=\xi^{y} \partial_{y}$. Then $\mathcal{L}_{\xi} \hat{g}=0$ implies that $\hat{g}$ is independent on $y$ and the null condition on $\xi$ implies

$$
\hat{g}_{y y}=0 .
$$

To proceed further we introduce suitable ansatz for vierbein, following $[12,13]$. We split 11 tangent space indices into $a=1, \ldots, 9$ and the remaining as,+- so that $\eta_{a b}=\delta_{a b}, \eta_{+-}=\eta_{-+}=1$. Then we have the following ansatz for $\hat{E}_{M}{ }^{A}$ :

$\hat{E}_{\mu}{ }^{a}=e_{\mu}{ }^{a}, \quad \hat{E}_{\mu}{ }^{-}=S^{-1} \tau_{\mu}, \quad \hat{E}_{\mu}{ }^{+}=-S m_{\mu}$,

$\hat{E}_{y}{ }^{a}=0, \quad \hat{E}_{y}{ }^{-}=0, \quad \hat{E}_{y}{ }^{+}=S$,

and hence we have the following components of the metric ${ }^{4}$ :

$\hat{g}_{\mu \nu}=h_{\mu \nu}-m_{\mu} \tau_{\nu}-\tau_{\mu} m_{\nu}, \quad \hat{g}_{\mu y}=\tau_{\mu}=\hat{g}_{y \mu}, \quad \hat{g}_{y y}=0$.

Let us now consider induced metric on the world volume of the M5-brane with world-volume coordinates $m, n=$ $0,1, \ldots, 5$ when we presume that M5-brane is extended along $y$-direction. Explicitly

$$
\xi^{5}=y
$$

so that the induced metric $\hat{g}_{m n}$ has the form

$\hat{g}_{\alpha \beta}=h_{\alpha \beta}-m_{\alpha} \tau_{\beta}-\tau_{\alpha} m_{\beta} \equiv \bar{h}_{\alpha \beta}, \quad \hat{g}_{y y}=0, \quad \hat{g}_{y \alpha}=\tau_{\alpha}$,

where $h_{\alpha \beta}=h_{\mu \nu} \partial_{\alpha} X^{\mu} \partial_{\beta} X^{\nu}, m_{\alpha}=m_{\mu} \partial_{\alpha} X^{\mu}, \tau_{\alpha}=\tau_{\mu} \partial_{\alpha} X^{\mu}$. For further purposes we also write elements of the worldvolume inverse metric in the form

$\hat{g}^{y y}=2 \Phi, \quad \hat{g}^{\alpha y}=-\hat{v}^{\alpha}, \quad \hat{g}^{y \alpha}=-\hat{v}^{\alpha}, \quad \hat{g}^{\alpha \beta}=h^{\alpha \beta}$,

where we defined

$\hat{v}^{\alpha}=v^{\alpha}-h^{\alpha \beta} m_{\beta}, \quad \Phi=-v^{\alpha} m_{\alpha}+\frac{1}{2} m_{\alpha} h^{\alpha \beta} m_{\beta}$,

and where we also introduced $v^{\alpha}, h^{\alpha \beta}$ through the relations

$$
\begin{aligned}
\tau_{\alpha} v^{\alpha}=-1, \quad \tau_{\alpha} h^{\alpha \beta} & =0, \quad v^{\alpha} h_{\alpha \beta}=0, \\
h_{\alpha \beta} h^{\beta \gamma}-\tau_{\alpha} v^{\gamma}- & =\delta_{\alpha}^{\gamma} .
\end{aligned}
$$

It is important to stress that $v^{\alpha}$ and $h^{\alpha \beta}$ are five dimensional vector and tensor respectively.

Let us now proceed to the null dimensional reduction of M5-brane action (1). First of all we again use the fact that generally we have

\footnotetext{
${ }^{4}$ Before we proceed further we would like to stress that it is not completely clear which ansatz we should use for null dimensional reduction of three form $C^{(3)}$. We expect that it could have the same form as in the case of spatial dimensional reduction and this is currently under study. Since no definite result has been derived yet we consider the case of zero three form field $C^{(3)}$ in this paper.
} 


$$
-\operatorname{det}\left(\hat{g}_{m n}+i \frac{\hat{H}_{m n}^{*}}{\sqrt{\partial \widehat{\partial \partial a} a}}\right)=-\operatorname{det} \hat{g}_{m r}\left(-\operatorname{det}\left(\hat{g}^{r s}+i \frac{1}{6 \sqrt{\widehat{\partial a \partial} a} \sqrt{-\hat{g}}} \epsilon^{r s t u v w} H_{t u v} \partial_{w} a\right)\right)\left(-\operatorname{det} \hat{g}_{s n}\right) .
$$

As in the previous section we find that nonzero components of $H_{m n r}$ are $H_{\alpha \beta \gamma}$ when we also imposed the gauge fixing condition (12). Using these facts we find that the second bracket in (49) is equal to

$$
\begin{aligned}
-\operatorname{det}\left(\hat{g}^{r s}+i \frac{1}{6 \sqrt{\widehat{\partial a \partial} a} \sqrt{-\hat{g}}} \epsilon^{\alpha \beta \gamma \delta \omega} H_{\gamma \delta \omega}\right) & =-\left|\begin{array}{cc}
h^{\alpha \beta}+i \frac{1}{6 \sqrt{2 \Phi} \sqrt{-\hat{g}}} \epsilon^{\alpha \beta \gamma \delta \omega} H_{\gamma \delta \omega} & -\hat{v}^{\beta} \\
-\hat{v}^{\alpha} & 2 \Phi
\end{array}\right| \\
& =-2 \Phi \operatorname{det}\left(h^{\alpha \beta}-\frac{1}{2 \Phi} \hat{v}^{\alpha} \hat{v}^{\beta}+i \frac{1}{6 \sqrt{-\operatorname{det} \bar{h}_{\alpha \beta}}} \epsilon^{\alpha \beta \gamma \delta \omega} H_{\gamma \delta \omega}\right),
\end{aligned}
$$

where in the final step we used the fact that

$$
\operatorname{det} \hat{g}_{m n}=\left|\begin{array}{cc}
0 & -\tau_{\beta} \\
-\tau_{\alpha} & \bar{h}_{\alpha \beta}
\end{array}\right|=\left|\begin{array}{cc}
-\tau_{\gamma} \bar{h}^{\gamma \delta} \tau_{\delta} & 0 \\
-\tau_{\alpha} & \bar{h}_{\alpha \beta}
\end{array}\right|=\frac{1}{2 \Phi} \operatorname{det} \bar{h}_{\alpha \beta}
$$

together with the fact that the matrix

$$
\bar{h}^{\alpha \beta}=h^{\alpha \beta}-\frac{1}{2 \Phi} \hat{v}^{\alpha} \hat{v}^{\beta}
$$

is inverse to $\bar{h}_{\alpha \beta}=h_{\alpha \beta}-m_{\alpha} \tau_{\beta}-\tau_{\alpha} m_{\beta}$ :

$$
\bar{h}_{\alpha \beta} \bar{h}^{\beta \gamma}=\delta_{\alpha}^{\gamma}
$$

This can be easily proved using the fact that

$$
\bar{h}_{\alpha \beta} \hat{v}^{\beta}=2 \tau_{\alpha} \Phi, \quad \tau_{\alpha} \bar{h}^{\alpha \beta} \tau_{\beta}=-\frac{1}{2 \Phi} .
$$

With the help of these results we obtain that the gauge fixed form of the expression (50) is equal to

$$
-\operatorname{det}\left(\hat{g}_{m n}+i \frac{\hat{H}_{m n}^{*}}{\sqrt{\widehat{\partial a \partial a}}}\right)=-\frac{1}{2 \Phi} \operatorname{det}\left(\bar{h}_{\alpha \beta}+i \frac{1}{6 \sqrt{-\operatorname{det} \bar{h}_{\alpha \beta}}} \bar{h}_{\alpha \gamma} \bar{h}_{\beta \delta} \epsilon^{\gamma \delta \sigma \omega \rho} H_{\sigma \omega \rho}\right) .
$$

Further we have

$$
-\frac{\sqrt{-\hat{g}}}{4 \widehat{\partial a \partial a}} \hat{H}^{* m n} H_{m n r} \partial^{r} a=\frac{1}{48 \Phi} \epsilon^{\alpha \beta \gamma \delta \omega} H_{\gamma \delta \omega} H_{\alpha \beta \sigma} \hat{v}^{\sigma}
$$

so that we obtain M5-brane action dimensionally reduced along the null direction in the form

$$
\begin{aligned}
S= & -T_{M 5} \int d y \int d^{5} \xi\left[\sqrt{-\frac{1}{2 \Phi} \operatorname{det}\left(\bar{h}_{\alpha \beta}+i \frac{1}{6 \sqrt{-\operatorname{det} \bar{h}_{\alpha \beta}}} \bar{h}_{\alpha \gamma} \bar{h}_{\beta \delta} \epsilon^{\gamma \delta \sigma \omega \rho} H_{\sigma \omega \rho}\right)}\right. \\
& \left.+\frac{1}{48 \Phi} \epsilon^{\alpha \beta \gamma \delta \omega} H_{\gamma \delta \omega} H_{\alpha \beta \sigma} \hat{v}^{\sigma}\right] .
\end{aligned}
$$


It is important to stress that $\Phi$ is equal to $-\frac{1}{2} \hat{v}^{\alpha} \bar{h}_{\alpha \beta} \hat{v}^{\beta}$. However it is convenient to treat it as independent when we add $\int d^{5} \xi B\left(2 \Phi+\hat{v}^{\alpha} \bar{h}_{\alpha \beta} \hat{v}^{\beta}\right)$ into action. Further, this action still depends on two form $B_{\alpha \beta}$ however it is more natural to find its dual formulation in the same way as in the case of the spatial dimensional reduction. In order to find such formulation we again extend the action as in the previous section,

$$
\begin{aligned}
S= & -T_{N D 4} \int d^{5} \xi\left[\sqrt{-\frac{1}{2 \Phi} \operatorname{det}\left(\bar{h}_{\alpha \beta}+i \frac{1}{6 \sqrt{-\operatorname{det} \bar{h}_{\alpha \beta}}} \bar{h}_{\alpha \gamma} \bar{h}_{\beta \delta} \epsilon^{\gamma \delta \sigma \omega \rho} H_{\sigma \omega \rho}\right)}\right. \\
& \left.+\frac{1}{48 \Phi} \epsilon^{\alpha \beta \gamma \delta \omega} H_{\gamma \delta \omega} H_{\alpha \beta \sigma} \hat{v}^{\sigma}+B\left(2 \Phi+\hat{v}^{\alpha} \bar{h}_{\alpha \beta} \hat{v}^{\beta}\right)\right] \\
& +\frac{1}{6} \int d^{5} \xi V^{\alpha \beta \gamma}\left(H_{\alpha \beta \gamma}-\left(\partial_{\alpha} B_{\beta \gamma}+\partial_{\beta} B_{\gamma \alpha}+\partial_{\gamma} B_{\alpha \beta}\right)\right),
\end{aligned}
$$

where we introduced effective nonrelativistic D4-brane tension through the relation

$$
T_{N D 4}=T_{M 5} \int d y
$$

In (58) we treat $H_{\alpha \beta \gamma}$ and $B_{\alpha \beta}$ as independent fields. Then the equations of motion for $B_{\alpha \beta}$ imply that $V^{\alpha \beta \gamma}$ is equal to

$$
V^{\alpha \beta \gamma}=\epsilon^{\alpha \beta \gamma \delta \omega} \partial_{\delta} A_{\omega} .
$$

Finally we should solve equations of motion for $H_{\alpha \beta \gamma}$. Since it is difficult to solve them in the full generality we proceed in the similar way as in the previous section even if we have to be more careful now. First of all we can still presume that the space metric $h_{\alpha \beta}$ has the form

$$
h_{\alpha \beta}=\delta_{\alpha}^{i} \delta_{\beta}^{j} \delta_{i j}, \quad i, j=1, \ldots, 4
$$

On the other hand, in the case of time one form we presume that it is constant and equal to

$$
\tau_{\alpha}=\tau \delta_{\alpha}^{0}
$$

We further presume that $m$ has nonzero components $m_{0}, m_{1}$ so that $\bar{h}_{00}=-2 \tau m_{0}, \bar{h}_{01}=-\tau m_{1}=\bar{h}_{10}$. Then since $v^{\alpha}$ is defined as $\tau_{\alpha} v^{\alpha}=-1$ we have $v^{0}=-\frac{1}{\tau}$. Finally, as in the previous section we presume that nonzero components of $H_{\alpha \beta \gamma}$ are $H_{340} \equiv H_{2}, H_{120} \equiv H_{1}$. Then the matrix $M_{\alpha \beta}=$ $\bar{h}_{\alpha \beta}+i \frac{1}{6 \sqrt{-\operatorname{det} \bar{h}_{\alpha \beta}}} \bar{h}_{\alpha \gamma} \bar{h}_{\beta \delta} \gamma^{\gamma \delta \sigma \omega \rho} H_{\sigma \omega \rho}$ has the form

$$
\left(\begin{array}{ccccc}
\bar{h}_{00} & \bar{h}_{01} & i \frac{\bar{h}_{01}}{\sqrt{-\bar{h}}} H_{2} & 0 & 0 \\
\bar{h}_{10} & 1 & i \frac{1}{\sqrt{-\bar{h}}} H_{2} & 0 & 0 \\
-i \frac{\bar{h}_{01}}{\sqrt{-\bar{h}}} H_{2} & -i \frac{1}{\sqrt{-\bar{h}}} H_{2} & 1 & 0 & 0 \\
0 & 0 & 0 & 1 & i \frac{1}{\sqrt{-\bar{h}}} H_{1} \\
0 & 0 & 0 & -i \frac{1}{\sqrt{-\bar{h}}} H_{1} & 1
\end{array}\right)
$$

where we defined $\bar{h}=\operatorname{det} \bar{h}_{\alpha \beta}=\bar{h}_{00}-\bar{h}_{01}^{2}$. Then the extended action (58) has the form

$$
\begin{aligned}
S= & -T_{N D 4} \int d^{5} \xi \sqrt{-\frac{\bar{h}}{2 \Phi}}\left[\sqrt{\left(1-\tilde{H}_{1}^{2}\right)\left(1-\tilde{H}_{2}^{2}\right)}+\sqrt{-\frac{\bar{h}\left(v^{0}\right)^{2}}{2 \Phi}} \tilde{H}_{1} \tilde{H}_{2}-\tilde{V}_{1} \tilde{H}_{1}-\tilde{V}_{2} \tilde{H}_{2}\right] \\
& +T_{N D 4} \int d^{5} \xi B\left(2 \Phi+2 \frac{m_{0}}{\tau}+m_{1}^{2}\right),
\end{aligned}
$$

where $V_{1} \equiv V^{120}, V_{2} \equiv V^{340}$ and where we performed rescaling $H_{1,2}=\sqrt{-\bar{h}} \tilde{H}_{1,2}, V_{1,2}=\frac{1}{\sqrt{2 \Phi}} \tilde{V}_{1,2}$. From (64) we obtain 
the following equations of motion:

$$
-\tilde{H}_{1} \sqrt{\frac{1-\tilde{H}_{2}^{2}}{1-\tilde{H}_{1}^{2}}}+\Delta \tilde{H}_{2}-\tilde{V}_{1}=0,-\tilde{H}_{2} \sqrt{\frac{1-\tilde{H}_{1}^{2}}{1-\tilde{H}_{2}^{2}}}+\Delta \tilde{H}_{1}-\tilde{V}_{2}=0,
$$

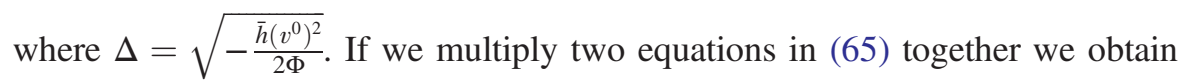

$$
\tilde{H}_{1} \tilde{H}_{2}=\left(\tilde{V}_{1}-\Delta \tilde{H}_{2}\right)\left(\tilde{V}_{2}-\Delta \tilde{H}_{1}\right)
$$

which allows us to express $\tilde{H}_{2}$ as

$$
\tilde{H}_{2}=\frac{\tilde{V}_{1} \tilde{V}_{2}-\Delta \tilde{H}_{1} \tilde{V}_{1}}{\left(1-\Delta^{2}\right) \tilde{H}_{1}+\Delta \tilde{V}_{2}}
$$

Inserting this result into the first equation in (65) we obtain the quadratic equation for $\tilde{H}_{1}$ that has the solution

$$
\tilde{H}_{1}=\frac{1}{1-\Delta^{2}}\left[-\Delta \tilde{V}_{2} \pm \tilde{V}_{1} \sqrt{\frac{\left(1-\Delta^{2}\right)+\tilde{V}_{2}^{2}}{\left(1-\Delta^{2}\right)+\tilde{V}_{1}^{2}}}\right]
$$

Then inserting this result into (67) we also find $\tilde{H}_{2}$ to be equal to

$$
\tilde{H}_{2}=\frac{1}{1-\Delta^{2}}\left[-\Delta \tilde{V}_{1} \pm \tilde{V}_{2} \sqrt{\frac{\left(1-\Delta^{2}\right)+\tilde{V}_{1}^{2}}{\left(1-\Delta^{2}\right)+\tilde{V}_{2}^{2}}}\right]
$$

Finally inserting (68) and (69) into the action (64) we obtain

$$
S=-T_{N D 4} \int d^{5} \xi\left[\sqrt{-\frac{1}{2 \Phi}\left(\bar{h}+\bar{h} \hat{V}_{1}^{2}\right)\left(1+\hat{V}_{2}^{2}\right)}-\frac{1}{2 \Phi} \bar{h} v^{0} \hat{V}_{1} \hat{V}_{2}+B\left(2 \Phi+2 \frac{m_{0}}{\tau}+m_{1}^{2}\right)\right],
$$

where we performed rescaling $\tilde{V}_{1.2}=\sqrt{1-\Delta^{2}} \hat{V}_{1,2}$ and where we have chosen a solution with a + sign in (68) and (69) in order to get an action with correct sign. To proceed further let us observe that we can write

$$
\bar{h}\left(1+\hat{V}_{1}^{2}\right)=\left|\begin{array}{ccc}
\bar{h}_{00}-\bar{h}_{01} \bar{h}_{10} & 0 & 0 \\
0 & 1 & \hat{V}_{1} \\
0 & -\hat{V}_{1} & 1
\end{array}\right|
$$

so that it is natural to introduce a redefined form of the metric $\bar{h}_{00}^{\prime}=\bar{h}_{00}-\bar{h}_{01} \bar{h}_{10}$. Further, we have $\bar{h} v^{0}=m_{0}+$ $m_{1} m_{0} \equiv m_{0}^{\prime}$ introducing redefined $m_{0}^{\prime}$. Using these results we can restore the general form of the action for the D4-brane in the Newton-Cartan background in the form

$$
S=-T_{N D 4} \int d^{5} \xi\left[\sqrt{-\frac{1}{2 \Phi}\left(\bar{h}_{\alpha \beta}+F_{\alpha \beta}\right)}-\frac{1}{16 \Phi} \epsilon^{\alpha \beta \gamma \delta \omega} m_{\alpha} F_{\beta \gamma} F_{\delta \omega}+B\left(2 \Phi+\hat{v}^{\alpha} \bar{h}_{\alpha \beta} \hat{v}^{\beta}\right)\right] .
$$

Finally we can integrate $B$ to replace $2 \Phi$ with $-\hat{v}^{\alpha} \bar{h}_{\alpha \beta} \hat{v}^{\beta}$. Then we can rewrite the action into an alternative form with the help of the following manipulation:

$$
\begin{aligned}
\frac{1}{2 \Phi} \operatorname{det}\left(\bar{h}_{\alpha \beta}+F_{\alpha \beta}\right) & =\frac{1}{2 \Phi} \operatorname{det} \bar{h}_{\alpha \gamma} \operatorname{det}\left(\delta_{\beta}^{\gamma}+\bar{h}^{\gamma \omega} F_{\omega \beta}\right) \\
& =\operatorname{det}\left(H_{\alpha \gamma}\right) \operatorname{det}\left(\delta_{\beta}^{\gamma}+\bar{h}^{\gamma \omega} F_{\omega \beta}\right)=\operatorname{det}\left(H_{\alpha \beta}+H_{\alpha \gamma} \bar{h}^{\gamma \omega} F_{\omega \beta}\right) \\
& =\operatorname{det}\left(H_{\alpha \beta}+\tilde{F}_{\alpha \beta}\right),
\end{aligned}
$$


where we introduced $H_{\alpha \beta}$ and its inverse $H^{\alpha \beta}$ as

$$
H_{\alpha \beta}=-\tau_{\alpha} \tau_{\beta}+h_{\alpha \beta}, \quad H^{\alpha \beta}=h^{\alpha \beta}-v^{\alpha} v^{\beta}
$$

and also $\tilde{F}_{\alpha \beta}$ through the relation

$$
\tilde{F}_{\alpha \beta}=H_{\alpha \gamma} \bar{h}^{\gamma \delta} F_{\delta \beta}
$$

Finally we used the fact that

$$
\begin{aligned}
\frac{1}{2 \Phi} \operatorname{det} \bar{h}_{\alpha \beta} & =\operatorname{det}\left(\begin{array}{cc}
0 & -\tau_{\beta} \\
-\tau_{\alpha} & \bar{h}_{\alpha \beta}
\end{array}\right)=\operatorname{det}\left(\begin{array}{cc}
0 & -\tau_{\beta} \\
-\tau_{\alpha} & -\tau_{\alpha} \tau_{\beta}+h_{\alpha \beta}
\end{array}\right) \\
& =\operatorname{det}\left(\begin{array}{cc}
-\tau_{\gamma} H^{\gamma \delta} \tau_{\delta} & 0 \\
-\tau_{\alpha} & H_{\gamma \delta}
\end{array}\right)=\operatorname{det}\left(H_{\alpha \beta}\right) .
\end{aligned}
$$

Then we obtain that the action (72) can be rewritten into the form

$$
\begin{aligned}
S= & -T_{N D 4} \int d^{5} \xi\left[\sqrt{-\operatorname{det}\left(H_{\alpha \beta}+\tilde{F}_{\alpha \beta}\right)}-\frac{1}{16 \Phi} \epsilon^{\alpha \beta \gamma \delta \omega} m_{\alpha} \tilde{F}_{\beta \gamma} \tilde{F}_{\delta \omega}\right. \\
& \left.-\frac{1}{8 \Phi} \epsilon^{\alpha \beta \gamma \delta \omega} m_{\alpha} \tilde{F}_{\beta \gamma} \tau_{\delta}\left(-v^{\sigma} \tilde{F}_{\sigma \omega}+\frac{1}{2 \Phi}\left(1+v^{\delta} m_{\delta}\right) \hat{v}^{\sigma} \tilde{F}_{\sigma \omega}\right)\right] .
\end{aligned}
$$

The action (72) or its alternative form (77) is the final form of the nonrelativistic D4-brane action in the background that arises by null dimensional reduction of M-theory. The kinetic part of the action has standard DBI like form and has functionally the same form as D2-brane action in torsional Newton-Cartan background that was found in [18]. However this result raises many questions. The most important one is that the D4-brane action has relativistic form even if it describes the D4-brane in the torsional nonrelativistic background. In fact, if we consider flat nonrelativistic space-time with $\tau_{\mu}=\delta_{\mu}^{0}, h_{\mu \nu}=\delta_{i j}$, $i, j=1, \ldots, 9$ together with $m_{\mu}=0$ and zero gauge field we get that the action reduces into manifestly Lorentz invariant form $S=-T_{N D 4} \int d^{5} \xi \sqrt{-\operatorname{det} \partial_{\alpha} x^{\mu} \partial_{\beta} x^{\nu} \eta_{\mu \nu}}$, $\eta_{\mu \nu}=\operatorname{diag}(-1,1, \ldots, 1)$. This unsolved problem is even more severe when we compare (72) with the form of nonrelativistic D4-brane in the torsional NC background as was found in [20,21]. In these papers the nonrelativistic Dp-brane in torsional NC background was derived from the relativistic $\mathrm{D}(p+1)$-brane that is extended along null isometry by T-duality. In fact, we could expect that following two chains of dimensional reductions and T-duality transformations should be related in some way. In the first one we perform null dimensional reduction of the M5-brane that leads to the action given in (72). Then performing T-duality along the longitudinal spatial dimension we get the D3-brane in T-dual theory. The second chain corresponds to spatial dimensional reduction of the M5-brane that leads to the relativistic D4-brane. Then performing T-duality along the null direction we obtain nonrelativistic D3-brane action as was found in [20,21]. At present it is not completely clear how these duality transformations are related.

The second line in (72) contains an additional mysterious term that has an exceptional property that it depends on the world-volume fields $v^{\alpha}, h^{\alpha \beta}$ and which does not have its analog in nonrelativistic action found in [20,21]. We are not able to explain the origin of this term in the action and certainly it would be very desirable to find its meaning.

\section{M5-BRANE TRANSVERSE TO NULL DIRECTION}

In this last section we briefly discuss the situation when we presume that the M5-brane is transverse to the null direction. In this case the induced metric has the form

$$
\begin{aligned}
& \hat{g}_{m n}=\bar{h}_{m n}+\tau_{m} \partial_{n} y+\partial_{m} y \tau_{n}, \quad \tau_{m}=\tau_{\mu} \partial_{m} X^{\mu}, \\
& \bar{h}_{m n}=\bar{h}_{\mu \nu} \partial_{m} X^{\mu} \partial_{n} X^{\nu},
\end{aligned}
$$

where

$$
\bar{h}_{m n}=h_{m n}-\tau_{m} m_{n}-\tau_{n} m_{m} .
$$

Note that this metric is nonsingular with inverse metric

$$
\bar{h}^{m n}=h^{m n}-\frac{1}{2 \Phi} \hat{v}^{m} \hat{v}^{n},
$$

where again 
$\hat{v}^{m}=v^{m}-h^{m n} m_{n}, \quad \Phi=-v^{m} m_{m}+\frac{1}{2} m_{m} h^{m n} m_{n}$,

where $v^{m}$ is a six dimensional vector that obeys $v^{m} \tau_{m}=-1, v^{m} h_{m n}=0$. Finally $h^{m n}$ is a six dimensional singular matrix that obeys

$$
h_{m n} h^{n k}-\tau_{m} v^{k}=\delta_{m}^{k}, \quad \tau_{m} h^{m n}=0 .
$$

In order to find the NS5-brane in torsional Newton-Cartan background it is appropriate to express all components of world-volume metric $\hat{g}_{m n}$ and its inverse $\hat{g}^{m n}$ with the help of $\bar{h}_{m n}, \tau_{m}, m_{n}$, derivatives of $y$ together with inverse fields $\bar{h}^{m n}, \hat{v}^{m}$. In the case of $\hat{g}_{m n}$ these relations are given in (78). In the case of $\hat{g}^{m n}$ the situation is more complicated and after some calculations we find

$$
\begin{aligned}
\hat{g}^{k l}= & \bar{h}^{k l}+\frac{1}{1+\frac{1}{\Phi} \hat{v}^{m} \partial_{m} y+\frac{1}{2 \Phi} \partial_{m} y h^{m n} \partial_{n} y}\left(-\frac{1}{4 \Phi^{2}} \hat{v}^{k} \hat{v}^{l} \partial_{m} y \bar{h}^{m n} \partial_{n} y\right. \\
& \left.+\frac{1}{2 \Phi} \bar{h}^{k p} \partial_{p} y\left(1+\frac{1}{2 \Phi} \hat{v}^{m} \partial_{m} y\right) \hat{v}^{l}+\frac{1}{2 \Phi} \hat{v}^{k}\left(1+\frac{1}{2 \Phi} \hat{v}^{m} \partial_{m} y\right) \partial_{p} y \bar{h}^{p l}-\frac{1}{2 \Phi} \bar{h}^{k m} \partial_{m} y \partial_{n} y \bar{h}^{n l}\right) .
\end{aligned}
$$

Then we obtain an action for the nonrelativistic NS5-brane when we insert (78) and (83) into (1). Note that there are two scalar fields $y(\xi), a(\xi)$ that propagate on the world volume of the NS5-brane. In fact this result is similar to transverse dimensional reduction of the M5-brane as was studied in [7]. However due to the form of the metric $\hat{g}_{m n}, \hat{g}^{m n}$ it is impossible to simplify M5-brane action further so that we will not write the explicit form of the action for the NS5-brane in torsional Newton-Cartan background here.

\section{ACKNOWLEDGMENTS}

This work is supported by the grant Integrable Deformations (GA20-04800S) from the Czech Science Foundation (GACR).
[1] E. Witten, String theory dynamics in various dimensions, Nucl. Phys. B443, 85 (1995).

[2] P. K. Townsend, D-branes from M-branes, Phys. Lett. B 373, 68 (1996).

[3] P. K. Townsend, Four lectures on M theory, arXiv:hep-th/ 9612121.

[4] P. Pasti, D. P. Sorokin, and M. Tonin, Covariant action for a $\mathrm{D}=11$ five-brane with the chiral field, Phys. Lett. B 398, 41 (1997).

[5] I. A. Bandos, K. Lechner, A. Nurmagambetov, P. Pasti, D. P. Sorokin, and M. Tonin, On the equivalence of different formulations of the M theory five-brane, Phys. Lett. B 408, 135 (1997).

[6] I. A. Bandos, K. Lechner, A. Nurmagambetov, P. Pasti, D. P. Sorokin, and M. Tonin, Covariant Action for the SuperfiveBrane of M Theory, Phys. Rev. Lett. 78, 4332 (1997).

[7] I. A. Bandos, A. Nurmagambetov, and D. P. Sorokin, The type IIA NS5-brane, Nucl. Phys. B586, 315 (2000).

[8] M. Aganagic, J. Park, C. Popescu, and J. H. Schwarz, Dual D-brane actions, Nucl. Phys. B496, 215 (1997).

[9] M. Aganagic, J. Park, C. Popescu, and J. H. Schwarz, World volume action of the M theory five-brane, Nucl. Phys. B496, 191 (1997).

[10] E. Witten, Five-brane effective action in M theory, J. Geom. Phys. 22, 103 (1997).
[11] D. Berman, M5 on a torus and the three-brane, Nucl. Phys. B533, 317 (1998).

[12] B. Julia and H. Nicolai, Null Killing vector dimensional reduction and Galilean geometrodynamics, Nucl. Phys. B439, 291 (1995).

[13] E. Bergshoeff, A. Chatzistavrakidis, L. Romano, and J. Rosseel, Newton-Cartan gravity and torsion, J. High Energy Phys. 10 (2017) 194.

[14] D. Hansen, J. Hartong, and N. A. Obers, Non-relativistic gravity and its coupling to matter, J. High Energy Phys. 06 (2020) 145.

[15] N. Lambert and T. Orchard, Null reductions of the M5-brane, J. High Energy Phys. 12 (2020) 037.

[16] C. D. A. Blair, D. Gallegos, and N. Zinnato, A nonrelativistic limit of M-theory and 11-dimensional membrane Newton-Cartan, J. High Energy Phys. 10 (2021) 015.

[17] J. Klusoň and P. Novosad, Non-relativistic M2-Brane, J. High Energy Phys. 06 (2019) 072.

[18] J. Kluson, Null dimensional reduction of M2-Brane, arXiv:2103.00566.

[19] A. A. Tseytlin, Selfduality of Born-Infeld action and Dirichlet three-brane of type IIB superstring theory, Nucl. Phys. B469, 51 (1996).

[20] J. Klusoň, Unstable D-brane in torsional Newton-Cartan background, J. High Energy Phys. 09 (2020) 191. 
[21] J. Gomis, Z. Yan, and M. Yu, T-duality in nonrelativistic open string theory, J. High Energy Phys. 02 (2021) 087.

[22] E. A. Bergshoeff, J. Gomis, J. Rosseel, C. Şimşek, and Z. Yan, String theory and string Newton-Cartan geometry, J. Phys. A 53, 014001 (2020).

[23] E. Bergshoeff, J. Gomis, and Z. Yan, Nonrelativistic string theory and T-duality, J. High Energy Phys. 11 (2018) 133.

[24] R. Andringa, E. Bergshoeff, J. Gomis, and M. de Roo, Stringy Newton-Cartan gravity, Classical Quant. Grav. 29, 235020 (2012).

[25] E. A. Bergshoeff, J. Lahnsteiner, L. Romano, J. Rosseel, and C. Şimşek, A non-relativistic limit of NS-NS gravity, J. High Energy Phys. 06 (2021) 021.

[26] J. Kluson, D-Brane actions in nonrelativistic string theory and T-duality, Phys. Rev. D 104, 086009 (2021).

[27] J. Kluson, Stable and unstable $\mathrm{D} p$-branes in $p$-brane Newton-Cartan background, J. Phys. A 54, 215401 (2021).
[28] J. Klusoň, Nonrelativistic string theory sigma model and its canonical formulation, Eur. Phys. J. C 79, 108 (2019).

[29] T. Harmark, J. Hartong, L. Menculini, N. A. Obers, and G. Oling, Relating non-relativistic string theories, J. High Energy Phys. 11 (2019) 071.

[30] T. Harmark, J. Hartong, L. Menculini, N. A. Obers, and Z. Yan, Strings with non-relativistic conformal symmetry and limits of the AdS/CFT correspondence, J. High Energy Phys. 11 (2018) 190.

[31] T. Harmark, J. Hartong, and N. A. Obers, Nonrelativistic strings and limits of the AdS/CFT correspondence, Phys. Rev. D 96, 086019 (2017).

[32] J. Klusoň, T-duality of non-relativistic string in torsional Newton-Cartan background, J. High Energy Phys. 05 (2020) 024.

[33] J. Klusoň, Remark about non-relativistic string in NewtonCartan background and null reduction, J. High Energy Phys. 05 (2018) 041. 